\title{
Acute kidney injury - improving outcomes with early risk assessment and intervention
}

\author{
N K Rathnamalala ${ }^{1}$, S Rajapakse ${ }^{2}$ \\ Journal of the Ceylon College of Physicians, 2014, 44, 22-27
}

\section{Summary}

Acute kidney injury (AKI) is being recognized around the world as a preventable and treatable cause of increasing morbidity and mortality. Epidemiological data from the developing world show an increasing trend in its incidence, though data from the developing world is still scarce. Causes for AKI differ among developed and developing countries with sepsis as the leading cause in the developed world while communicable diseases, toxin related acute kidney injuries as well as those associated with herbal medications still contribute to the incidence in the developing world. The mortality following AKI is between $20-60 \%$ with many needing intensive care unit (ICU) admissions and renal replacement therapy (RRT). There is increasing evidence that AKI may lead to chronic kidney disease (CKD). Early risk assessment and adoption of simple prevention strategies have shown to improve outcomes in AKI.

\section{Introduction}

Acute kidney injury ( $\mathrm{AKI})$ is emerging as an important cause of morbidity and mortality throughout the world. There is increasing evidence that even mild impairment of renal function can lead to serious consequences ${ }^{1}$. Possible explanations for this effect are volume overload, retention of uremic compounds, acidosis, electrolyte disorders, increased risk of infection and anaemia ${ }^{2}$.

\section{Background}

The first description of acute kidney injury was by William Heberden in 1802 where it was named "ischuria renalis"3. During the first world the syndrome was named "war nephritis" and again came in to light during the second world war following a publication on crush syndrome ${ }^{4}$. There have been several different definitions used in the literature until recently when two classification systems were introduced. More recently kidney disease improving global outcomes

\footnotetext{
${ }^{1}$ University Medical Unit, National Hospital of Sri Lanka.

${ }^{2}$ Department of Clinical Medicine, Faculty of Medicine, Colombo, Sri Lanka.
}

(KDIGO) made modifications to these classification systems and introduced a new classification system for $A K I^{5}$.

\section{Incidence and aetiologies}

Epidemiological data on the incidence of $A K I$ is mostly lacking. A recent publication by $\mathrm{Hsu}$ et al. revealed a $10 \%$ annual increase in dialysis requiring AKI in the United States. An adult cohort of beneficiaries of the health-care delivery system Kaiser Permanente of Northern California were evaluated over a 8-year period from 1996 till 2003, and found that the use of acute renal replacement therapy (RRT) increased from 195 patients per million population per year (pmp/y) in the period 1996-1997 to $295 \mathrm{pmp} / \mathrm{y}$ in the period 20022003. Between the years 1996-2003, there was also an increased incidence of $\mathrm{AKI}$ not requiring dialysis from 323 to 522 per 100000 person-years $^{6}$.

Etiologies for AKI differ among developed and developing countries. While sepsis remains the leading cause of $A K I$ in developed countries, in the developing world infections remain the leading cause for $A K I^{7,8}$. In sub-Saharan Africa diarrheal disease, malaria, nephrotoxic traditional medicines, post obstetric complications and HIV are the leading causes ${ }^{8,9}$. In Asia diarrheal disease, malaria, leptospirosis and snake bites constitute over $60 \%$ of $A K I^{8,10,11}$. There is little epidemiological data from Latin America, however communicable diseases remain an important cause ${ }^{12}$. ICU-specific data from developed countries indicates an incidence of hospital-acquired AKI of between $5 \%$ and $20 \%$ of ICU admissions, corresponding to $66-80$ per million population per year ${ }^{13,14,15}$.

Among infectious diseases causing AKI, malaria is an important cause. The incidence varies from $0.6 \%$ to $60 \%$ in various geographical regions ${ }^{16}$. Leptospirosis is a common cause of AKI in tropical and subtropical countries. It is the leading cause of AKI in Singapore and Thailand ${ }^{17}$. Acute glomerulo-nephritis (AGN) remains an important mechanism underlying $\mathrm{AKI}$ in the developing world. In areas such as southeast Anatolia in Turkey, AGN causes more than $60 \%$ of cases of $\mathrm{AKI}^{18}$. Herbal and non-herbal nephrotoxins are common causes of $\mathrm{AKI}$ in tropical regions including Africa. The toxicity of herbal products (e.g.Callilepis 
laureola, known by Zulus as 'impila') often leads to liver failure, neurologic abnormalities, metabolic acidosis, AKI or respiratory failure ${ }^{19,20}$.

\section{Prognosis}

Many studies on AKI have been on ICU patients and reported outcomes have been poor. In one study, ICU mortality was $52 \%$ with an overall mortality of $60.3 \%$. Independent risk factors for hospital mortality included use of vasopressors (odds ratio [OR], 1.95; 95\% Cl, 1.50-2.55; $\mathrm{P}<.001$ ), mechanical ventilation (OR, 2.11; 95\% Cl, 1.58-2.82; $\mathrm{P}<.001$ ), septic shock (OR, 1.36; 95\% Cl, 1.03-1.79; $\mathrm{P}=.03$ ), cardiogenic shock (OR, 1.41; 95\% Cl, 1.05-1.90; $\mathrm{P}=.02$ ), and hepatorenal syndrome $(\mathrm{OR}, 1.87 ; 95 \% \mathrm{Cl}, 1.07-3.28$; $\mathrm{P}=0.03)^{13}$. In another study the mortality ranged from $8.8 \%$ to $26.3 \%$ with increasing severity of AKI. Patients with maximum RIFLE class $R$, class $I$ and class $F$ had hospital mortality rates of $8.8 \%, 11.4 \%$ and $26.3 \%$, respectively, compared with $5.5 \%$ for patients without acute kidney injury. Additionally, acute kidney injury (hazard ratio, 1.7; 95\% confidence interval, 1.28-2.13; $\mathrm{P}<0.001$ ) and maximum RIFLE class I (hazard ratio, $1.4 ; 95 \%$ confidence interval, 1.02-1.88; $P=0.037$ ) and class $F$ (hazard ratio, 2.7; 95\% confidence interval, 2.03-3.55; $P<0.001$ ) were associated with hospital mortality after adjusting for multiple covariates ${ }^{21}$. A study by Ostermann and Chang showed a hospital mortality rate ranging between $20.9 \%$ to $56.8 \%$ compared to $8.4 \%$ among patients without acute kidney injury ${ }^{22}$.

In a study conducted with data from the Australian New Zealand Intensive Care Society Adult Patient Database in 57 ICUs across Australia, AKI, defined by any RIFLE category, was associated with an increase in hospital mortality (OR 3.29, 95\% Cl 3.19-3.41, P < $0.0001)$. The crude hospital mortality stratified by RIFLE category was $17.9 \%$ for Risk, $27.7 \%$ for injury and $33.2 \%$ for failure. By multivariable analysis, each RIFLE category was independently associated with hospital mortality (OR: Risk 1.58, injury 2.54 and failure $3.22)^{23}$.

There is also increasing evidence to support that persistent renal dysfunction or CKD may be associated with AKI. Several studies support this. In the large multicenter BEST Kidney trial, $13 \%$ of patients still required $R R T$ at hospital discharge ${ }^{13}$. Similar findings were reported in Sweden: $8.3 \%$ of patients on continuous RRT and $16.5 \%$ of patients on intermittent RRT developed end-stage kidney disease ${ }^{24}$. In Canada, $22 \%$ of surviving patients developed end stage kidney disease ${ }^{25}$. Finally, patients who already have chronic kidney disease are at much greater risk of developing end stage kidney disease. In the large Scottish database, $13 \%$ of patients with normal baseline kidney function developed end stage kidney disease, compared with $53 \%$ in patients who at baseline already had chronic kidney insufficiency ${ }^{26}$.

\section{Situation in Sri Lanka}

Though countrywide data is unavailable in Sri Lanka, a study $(n=68)$ conducted at the National Hospital of Sri Lanka showed that sepsis and leptospirosis accounted for the majority of AKI presentations. Causes for sepsis were largely unidentified but urinary tract infection was the leading cause among those that were identified. Fifty eight (85.3\%) patients required RRT with 46 (79.32\%) receiving hemo-dialysis and $12(20.68 \%)$ receiving acute peritoneal dialysis. Twenty patients (29.4\%) made a complete recovery while $28(41.2 \%)$ made partial recovery with a mortality of $24 \%$. Of the patients who made a complete recovery $70 \%$ were below the age of 55 compared to $30 \%$ who were over 55 ( $p=0.13$ ). The presence of co morbidities was associated with a worse outcome with only $5.9 \%$ achieving complete recovery $(p<0.01)^{27}$.

\section{Risk assessment}

There are many known exposures for AKI such as sepsis, critical illness, circulatory shock, burns, trauma etc; however the incidence varies even among individuals exposed to the same insult. Data from many large prospective studies have supported the presence of susceptibility factors for developing AKI.

\section{Dehydration/volume depletion}

Volume depletion is an important and reversible cause of AKI. This is accepted as a risk factor for development of $\mathrm{AKI}$ upon exposure to any insult. $\mathrm{A}$ systematic review of the literature to determine the ability of dynamic changes in arterial waveform-derived variables to predict fluid responsiveness and $56 \%$ of patients responded to a fluid challenge ${ }^{28}$. However, none of the existing guidelines have been designed to evaluate renal outcomes. Optimal fluid parameters and hemodynamic targets have not been established for AKI management ${ }^{29}$.

\section{Age}

Older age has been associated with the development of AKI both in hospital and in the community. The higher incidence in older age $(>65)$ has been demonstrated with many insults such as radiocontrast, carotid surgery and nephrotoxic drugs. In a populationbased surveillance conducted among all adult residents of the Calgary Health Region, admitted to multidisciplinary and cardiovascular surgical intensive care units between May 11999 and April 30 2002, the highest incidence of $\mathrm{AKI}$ was among older patients 
$(>65 y e a r s)^{25}$. In another study to identify and validate a risk prediction score for contrast induced nephropathy, age more than 75 years was an independent predictor of $\mathrm{AKI}$ in multivariate logistic regression with a $\mathrm{p}<0.0001^{30}$.

\section{Gender}

Many studies have shown a higher occurrence of AKI in hospitalized females. This also has been seen with many different exposures such as contrast and drugs $^{30,31}$. In a study to assess risk factors for developing $\mathrm{AKI}$ among patients undergoing cardiac surgery, of 32217 patients $1.7 \%$ developed AKI requiring dialysis. In the univariate comparison females were more likely to have AKI $p<0.001^{32}$. However, Males consistently predominated in reports on the incidence of AKI complicating infection with HIV, malaria, and leptospirosis ${ }^{33,34,35}$.

\section{Hypoalbuminemia}

Hypoalbuminemia has been identified as a risk factor for developing AKI. It has also been demonstrated to be a predictor of death following the development of AKI. With each $10 \mathrm{~g} \mathrm{~L}-1$ decrement in serum albumin the odds of AKI increased by $134 \%$ (pooled OR, 2.34; $\mathrm{Cl}, 1.74-3.14$ ). Among patients who had developed AKI, the odds of death rose $147 \%$ (pooled OR $2.47,95 \%$ $\mathrm{Cl}$ 1.51-4.05) with each $10 \mathrm{~g} \mathrm{~L}-1$ serum albumin decrement ${ }^{36}$.

\section{Chronic kidney disease (CKD)}

$C K D$ is the most consistent pre-existing condition associated with a high risk of AKI in almost every relevant study addressing hospital- or communityacquired $A K I^{37}$. Pre-existing CKD increases the risk of non recovery from $\mathrm{AKI}$. In a large, community based study in Northern California comprising 39,805 patients with CKD Stage 3B or higher, the risk for ESRD or death 30 days after hospital discharge was increased by $30 \%$ as a direct consequence of an episode of $A K{ }^{38}$.

\section{Diabetes mellitus}

Diabetes mellitus is well-known for its deleterious effect of AKI superimposed on CKD. In a study undertaken in India, histological findings in patients with AKI showed underlying diabetic changes. In addition 27\% had superimposed non diabetic changes responsible for AKI with diffuse proliferative changes being the commonest ${ }^{39}$. Diabetes mellitus is reported to be an important risk factor for AKI in hospital setting, particularly in association with cardiac surgery or exposure to radiocontrast media ${ }^{37}$. Diabetes mellitus is a risk factor for poor 1-year outcomes, including ESRD and death ${ }^{25}$.

\section{Heart disease}

Cardiac dysfunction has been identified as an important risk factor for AKI. In a case control study among hospitalized patients in Cleveland Ohio, heart failure was associated with an OR of 1.4 (Cl 0.6-2.4) in multivariate analysis ${ }^{40}$. It is also associated with a higher incidence of induced $A K{ }^{30}$.

\section{Pre exposure medication}

There is contrasting evidence on the impact of ACE inhibitors/ARBs on AKI. In a retrospective cohort study of 1358 adult patients undergoing cardiac surgery, it was found that preoperative use of ACE-I or angiotensin receptor blockers (ARB) was associated with a $27.6 \%$ higher risk for AKI postoperatively ${ }^{41}$. A propensity score-based analysis of 536 patients undergoing coronary artery bypass graft on cardiopulmonary bypass was conducted. In this study, the incidence of AKI was $6.4 \%$ in patients who received preoperative ACE inhibitors and $12.2 \%$ in patients who did not $(P<0.02)^{42}$. In a study by Kiski et al. patients treated with renin-angiotensin aldosterone system blockade before exposure to radiocontrast agents developed significantly more contrast induced acute kidney injury (Cl-AKI) within 72 hours and 30 days. Even after adjustment for confounding comorbidities, treatment with ACE-I or angiotensin II receptor type 1 blockers turned out to be an independent risk predictor $^{43}$.

\section{Specific exposures among acute medical admission}

\section{Nephrotoxic medication}

The risk of AKI attributable to aminoglycosides is sufficiently high and varies with the definitions used for AKI and the study populations $s^{44,45,46}$. Some authors have called for elimination of amino glycosides from the standard antimicrobial agents for and use only for directed treatment of a number of severe gram positive and negative bacterial infections ${ }^{47}$. Older patients with pre existing renal dysfunction, septic patients with intravascular volume depletion may be at greater risk of aminoglycoside nephrotoxicity. Concomitant use of other nephrotoxic drugs, prolonged use and separate courses of aminoglycoside therapy over short time intervals also increase the risk of $\mathrm{AK}^{48}$.

\section{Contrast induced nephropathy}

Contrast induced nephropathy (CIN) is defined as a rise in serum creatinine of $>0.5 \mathrm{mg} / \mathrm{dl}$ or a $25 \%$ increase from baseline value assessed at $48 \mathrm{hrs}$ after a radiological procedure with contrast administration ${ }^{49}$. However it is also noted in the literature that the increase in serum creatinine may occur until up to 5 days after contrast exposure ${ }^{50}$. It is accepted that in patients with normal renal functions even in the 
presence of diabetes the risk of contrast induced AKI is low $(1-2 \%)^{51}$. However the incidence may be as high as $25 \%$ in patients with pre existing renal impairment or in the presence of risk factors such as CKD, diabetes, CHF, advanced age, and concurrent administration of nephrotoxic drugs ${ }^{52}$. Contrast induced AKI was described as the third most common cause of new AKI in hospitalized patients and was responsible for $11 \%$ of cases $^{53}$.

\section{Prevention of $\mathrm{AKI}$ and early intervention strategies}

Due to the heterogeneity of patients and the broad range of situations in which AKI is encountered, it is difficult to standardize the evaluation and management approach. The KDIGO clinical guidelines on the management of AKI provide a useful reference for this purpose ${ }^{5}$.

KDIGO guidelines suggest the following in the high risk population

- Discontinue all nephrotoxic agents

- Ensure volume status and perfusion pressure

- Consider functional hemo-dynamic monitoring

- Monitor serum creatinine and urine output

- Avoid hyperglycemia

- Consider alternatives to radio contrast procedures.

\section{Conclusion}

Acute kidney injury is a common problem in medical, surgical, oncology and ICU settings in hospital. There is increasing evidence that irrespective of its nature AKI is a predictor of immediate and long term adverse outcomes. Individuals with CKD and other co-morbidities are especially susceptible to AKI and also AKI may be an independent risk factor for developing CKD. Research over the past decade has identified numerous preventable risk factors for AKI with the potential of improving the management and outcomes. A significant proportion of in hospital AKI is due to pre renal causes which is potentially reversible. Identifying those at risk and also those that are more prone to residual dysfunction enables aggressive and timely management. This is particularly relevant to Sri Lanka where there is a scarcity of resources for renal replacement therapy.

\section{References}

1. Uchino S, Bellomo R, Goldsmith D, et al. An assessment of the RIFLE criteria for acute renal failure in hospitalized patients. Critical Care Medicine 2006; 34:1913-17.

2. Hoste EA, Kellum JA. Acute Renal Failure in the critically ill; impact on morbidity and mortality. Contributions to Nephrology 2004; 144: 1-11.

3. Eknoyan G. Emergence of the concept of acute renal failure. American Journal of Nephrology 2002; 22: 225- 30.

4. Bywaters EGL, Beall D. Crush injuries with impairment of renal functions. British Medical Journal 1947; 1:427-32.

5. Kidney Disease Improving Global Outcomes (KDIGO). Clinical Practice Guidelines for Acute Kidney Injury. Kidney International Supplements 2012; 2,19-36.

6. Hsu R K, McCulloch CE, Dudley RA, Lo LJ, Hsu C: Temporal Changes in Incidence of Dialysis-Requiring AKI. Journal of American Society of Nephrology 2013; 24: 37-42.

7. Ali T, Khan I, Simpson W, et al. Incidence and outcomes in acute kidney injury: a comprehensive population-based study. Journal of American Society of Nephrology 2007; 18: $1292-8$.

8. Cerda J, Bagga A, Kher V, et al. The contrasting characteristics of acute kidney injury in developed and developing countries. Nature Clinical Practice Nephrology 2008; 4: 138-53.

9. Naicker S, Aboud O, Gharbi MB. Epidemiology of acute kidney injury in Africa. Seminars in Nephrology 2008; 28 : 348-53.

10. Jha V, Chugh KS. Community-acquired acute kidney injury in Asia. Seminars in Nephrology 2008; 28: 330-47.

11. Jha V, Rathi M. Natural medicines causing acute kidney injury. Seminars in Nephrology 2008; 28: 416-28.

12. Lombardi R, Yu L, Younes-Ibrahim M, et al. Epidemiology of acute kidney injury in Latin America. Seminars in Nephrology 2008; 28: 320-9.

13. Uchino S, Bellomo R, Morimatsu S, et al. External validation of severity scoring systems for acute renal failure using a multinational database. Critical Care Medicine 2005; 33: 1961-7.

14. Metnitz PG, Krenn CG, Steltzer H, et al. Effect of acute renal failure requiring renal replacement therapy on outcome in critically ill patients. Critical Care Medicine 2002; 30: 2051-8.

15. Liano F,Junco E, Pascual J, Madero R,Verde E. The spectrum of acute renal failure in the intensive care unit compared with that seen in other settings. The Madrid Acute Renal Failure Study Group. Kidney International 1998; 66 (Suppl): S16-S24.

16. Mehta KS, Halankar Ar, Makwana PD, et al. Severe acute renal failure in malaria. Journal of Postgraduate Medicine 2001; 47: 24-6.

17. Sitprija V. Renal dysfunction in leptospirosis: a view from the tropics. Nature Clinical Practice Nephrology 2006; 2: 658-9. 
18. Bircan Z Kervancio lu M, Katar S, Onur A, Onur H et al. The etiology of renal failure in south-east Anatolia. Pediatric Nephrology 2000; 14: 87-8.

19. Kadiri S, Arije A,Salako B L. Traditional herbal preparations and acute renal failure in south west Nigeria. Tropical Doctor 1999; 29: 244-6.

20. Otieno LS, McLigeyo SO, Luta M. Acute renal failure following the use of herbal remedies. East African Medical Journal 1991 68: 993-8.

21. Hoste EA, Clermont G, Kersten A, et al. RIFLE criteria for acute kidney injury are associated woth hospital mortality in critically ill patients: a cohort analysis. Critical Care 2006; 10: R73.

22. Ostermann M, Chang RW. Acute kidney injury in the intensive care unit according to RIFLE. Critical Care Medicine 2007; 35: 1837-43.

23. Bagshaw SM, George C, Dinu I, et al. A multi centre evaluation of the RIFLE criteria for early acute kidney injury in critically ill patients. Nephrology Dialysis and Transplantation 2008; 23:1203-10.

24. Bell M, Granath F, Schon S, et al. Continuous renal replacement therapy is associated with less chronic renal failure than intermittent haemodialysis after acute renal failure. Intensive Care Medicine 2007; 33: 773-80.

25. Bagshaw SM, Laupland KB, Doig CJ, et al. Prognosis for long-term survival and renal recovery in critically ill patients with severe acute renal failure: $A$ population-based study. Critical Care 2005; 9: R700-R709.

26. Prescott GJ, Metcalfe W, Baharani J, et al. A prospective national study of acute renal failure treated with RRT: incidence, aetiology and outcomes. Nephrology Dialysis and Transplanation 2007; 22: 2513-19.

27. Rathnamalala N. Clinical characteristics and outcomes of acute kidney injury. Saudi Journal of Kidney Disease and Transplantation 2013 ; 24(4): 813-5.

28. Marik PE, Cavallazzi R, Vasu T, Hirani A. Dynamic changes in arterial waveform derived variables and fluid responsiveness in mechanically ventilated patients: a systematic review of the literature. Critical Care Medicine 2009; 37: 2642-7.

29. Glasmacher A, von Lilienfeld- Toal M, Sculte S, et al. An evidence based evaluation of important aspects of empirical antibiotic therapy in febrile neutropenic patients. Clinical Microbiology and infection 2005; 11(5): 17-23.

30. Mehran R, Aymong ED, Nikolsky E, et al. A simple risk score for prediction of contrast-induced nephropathy after percutaneous coronary intervention: development and initial validation. Journal of the American College of Cardiology 2004; 44: 1393-9.

31. Smith CR, Moore RD, Lietman PS. Studies of risk factors for aminoglycoside nephrotoxicity. American Journal of Kidney Disisease 1986; 8: 308-13.
32. Thakar CV, Liangos O, Yared JP, et al. ARF after open-heart surgery: Influence of gender and race. American Journal of Kidney Disease 2003; 41: 742 -51.

33. Cohen SD, Chawla LS, Kimmel PL. Acute kidney injury in patients with human immunodeficiency virus infection. Current Opinion in Critical Care 2008; 14: 647-53.

34. Abdul Manan J, Ali H, Lal M. Acute renal failure associated with malaria. Journal of Ayub Medical College Abbottabad 2006; 18: 47-52.

35. Ittyachen AM, Krishnapillai TV, Nair MC, et al. Retrospective study of severe cases of leptospirosis admitted in the intensive care unit. Journal of Postgraduate Medicine 2007; 53: $232-5$.

36. Wiedermann C, Weidwermann W, Joannidis M. Hypoalbuminemia and acute kidney injury: a meta-analysis of observational clinical studies. Intensive Care Medicine 2010; 36: 1657-65.

37. Rodrigo Cartin-Ceba, Markos Kashiouris, Maria Plataki et al. Risk Factors for Development of Acute Kidney Injury in Critically III Patients: ASystematic Review and Meta-Analysis of Observational Studies. Critical Care Research and Practice 2012; 10: 1151-60.

38. Hsu CY, Chertow GM, McCulloch CE, et al. Nonrecovery of kidney function and death after acute on chronic renal failure. Clinical Journal of American Society of Nephrology 2009; 4: 891-8.

39. Moger V, Kumar SK, Sakhuja V, et al. Rapidly progressive renal failure in type 2 diabetes in the tropical environment: a clinico-pathological study. Renal Failure 2005; 27: 595-600.

40. Drawz PE, Miller RT, Sehgal AR. Predicting hospital-acquired acute kidney injury - a case-controlled study. Renal Failure 2008; 30: 848-55.

41. Arora P, Rajagopalam S, Ranjan R, et al. Preoperative use of angiotensin-converting enzyme inhibitors/angiotensin receptor blockers is associated with increased risk for acute kidney injury after cardiovascular surgery. Clinical Journal of American Society of Nephrology 2008; 3: 1266-73.

42. Benedetto U, Sciarretta S, Roscitano A, et al. Preoperative Angiotensin-converting enzyme inhibitors and acute kidney injury after coronary artery bypass grafting. Annals of Thoracic Surgery 2008; 86: 1160-5.

43. Kiski D, Stepper W, Brand E, et al. Impact of reninangiotensin-aldosterone blockade by angiotensin converting enzyme inhibitors or AT-1 blockers on frequency of contrast medium-induced nephropathy: a post-hoc analysis from the Dialysis-versus-Diuresis (DVD) trial. Nephrology Dialysis and Transplantation 2010; 25: 759-64.

44. Bliziotis IA, Michalopoulos A, Kasiokou SK, et al. Ciprofloxacin vs an aminoglycoside in combination with a beta lactam for 
the treatment of bacterial endocarditis: a meta analysis of randomized controlled trials. Mayo Clinic Proceedings 2005; 80: $1146-56$.

45. Falgas Me, Maththiou DK, Bliziotis IA. The role of aminoglycosides in combination with a beta lactam for the treatment of bacterial endocarditis: a meta nanalysis of comparative trials. Journal of Antimicrobial Chemotherapy 2006; 57 : 639-47.

46. Godin M, Bouchard J, Mehta R. Fluid balance in patients with acute kidney injury: emerging concepts. Nephron Clinical Practice 2013; 123: 238-45.

47. English WP, Williams MD. Should aminoglycoside antibiotics be abandoned? American Journal of Surgery 2000; 180: 512-15.

48. Paul M, Benuri- Silbiger I Soares Weiser K, et al. beta lactam monotherapy versis beta lactam aminoglycoside combination therapy for sepsis. Cochrane Data Base systematic review 2006: CD003344.
49. Harjai KJ, Raizada A,Shenoy C, et al. A comparison of contemporary definitions of contrast nephropathy in patients undergoing percutaneous coronary intervention and a proposal for a novel nephropathy grading system. American Journal of Cardiology 2008; 101: 812- 19.

50. Ribichini F, Graziani M, Gambaro G, et al. Early creatinine shifts predict contrast induced nephropathy and persistnent renal damage after angiography. American Journal of Medicine 2010; 123: 755-63.

51. Berns A. Nephrotoxicity of contrast media. Kidney International 1989; 36: 730 -40.

52. Rudnick MR, Goldfarb S, Tumulin J. Contrast induced nephropathy: is the picture any clearer? Clinical Journal of the American Society of Nephrology 2008; 3: 261-2.

53. Nash K, Hafeez A, Hou S. Hospital acquired renal insufficiency. American Journal of Kidney Disease 2002; 39: 930-6. 